\title{
SI - A compact Incoherent Broadband Cavity Enhanced Absorption Spectrometer (IBBCEAS) for trace detection of nitrogen oxides, iodine oxide and glyoxal at sub-ppb levels for field application
}

Albane Barbero ${ }^{1}$, Camille Blouzon ${ }^{1}$, Joël Savarino ${ }^{1}$, Nicolas Caillon ${ }^{1}$, Aurélien Dommergue ${ }^{1}$, and Roberto Grilli ${ }^{1}$

${ }^{1}$ Univ. Grenoble Alpes, CNRS, IRD, Grenoble INP*, IGE, 38000 Grenoble, France

*Institute of Engineering Univ. Grenoble Alpes

Correspondence: Roberto Grilli (roberto.grilli@cnrs.fr)

1 Method

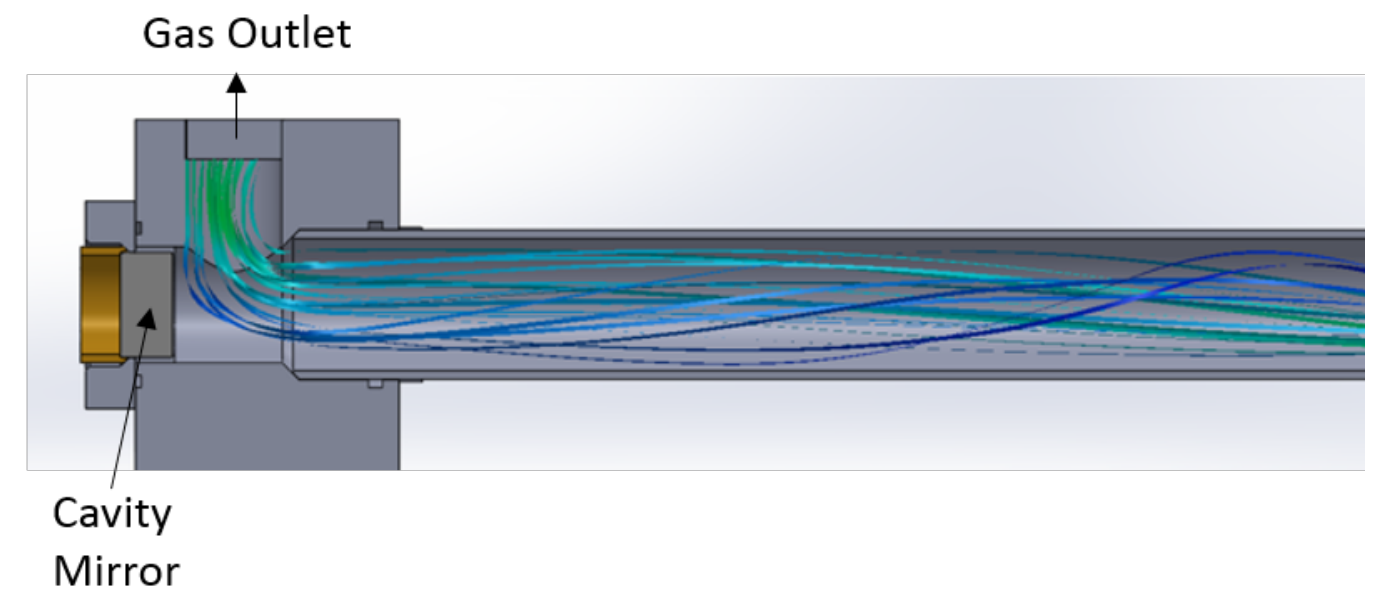

Figure SI - 1. SolidWorks Flow simulation of the air flow entering the cavity at $1 \mathrm{~L} \mathrm{~min}^{-1}$. The simulation allowed to place the gas outlet at a certain distance from the highly reflective mirrors in order to maximize the effective optical pathlength, avoiding the use of purging gas at the mirrors while preserving the mirror cleanliness during the measurement. The gas inlet is placed at the center of the cavity. 


\section{Spectral Fit}

For retrieving the wavelength dependent mirror reflectivity curve, literature spectra (from Vandaele 1998) was convoluted by the known instrumental function of the spectrometer $\left(18 \mathrm{~cm}^{-1}\right.$ Lorentzian broadening). The convoluted literature spectra is

5 then transformed into absorption coefficient (for a given $\mathrm{NO}_{2}$ concentration, in this case $1 \mathrm{ppm}$ ) by using the combination of equations 1 and 2 reported in the manuscript. It should be noticed that the trace gas concentration only plays on the mirror reflectivity curve offset and is not critical for determining its shape. As a first guess for the reflectivity curve, the theoretical values provided by the mirror manufacturer were use. The polynomial coefficients used to describe the mirror reflectivity curve were then slightly adjusted for achieving the best match between the convoluted literature spectrum and the measured spectrum (i.e. lower root-mean-square on the difference between the two data sets). In the Figure SI-2 the optimal mirror reflectivity curve is reported and defined by the fourth order polynomial equation reported as a function of the central wavelength $(\lambda-450 \mathrm{~nm})$ centering:

$$
\begin{aligned}
p(\lambda)=0.999913+8.42057 E^{-8}(\lambda-450 \mathrm{~nm}) & -1.15576 E^{-7}(\lambda-450 \mathrm{~nm})^{2} \\
+ & 2.21706 E^{-9}(\lambda-450 \mathrm{~nm})^{3}-1.95844 E^{-10}(\lambda-450 \mathrm{~nm})^{4}
\end{aligned}
$$

15 where $\lambda$ is the wavelength. At the bottom, the convoluted and unconvoluted literature spectrum from Vandaele 1998 is shown in unit of absorption coefficient, together with the acquired IBBCEAS spectrum. The blue line is the difference between the experimental and the convoluted literature spectra. Small discrepancies are visible which could be due to small anomalies in the mirror reflectivity curve that would require a larger order polynomial function for better matching, or to some experimental bias either on the literature or the IBBCEAS spectra (i.e. an inhomogeneous response of the pixels of the CCD camera or a bad baseline subtraction). Nevertheless, this does not represent an issue here since the reference spectra used for the fit analysis are the ones experimentally acquired by the same IBBCEAS instrument and calibrated against literature cross sections. 


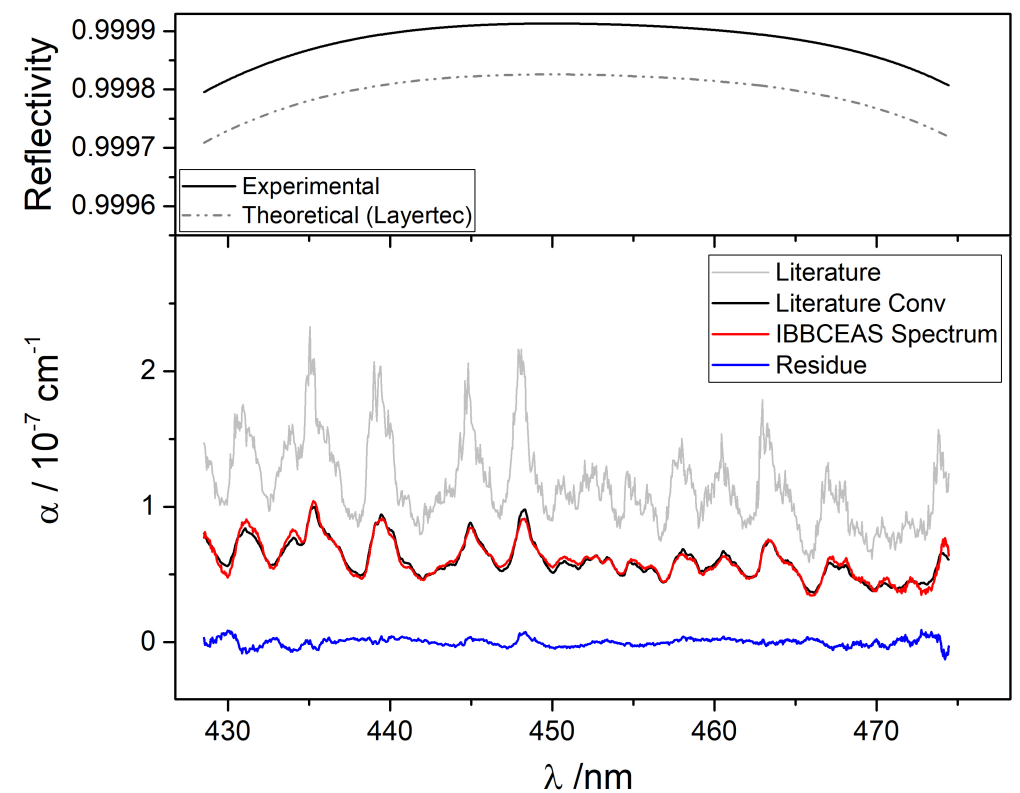

Figure SI - 2. (top) The optimal mirror reflectivity curve (well matching in shape the one provided by the manufacturer) which provides the best match between the literature and experimental spectra. (bottom) The convoluted and unconvoluted literature spectra (Vandaele 1998) and the experimental IBBCEAS spectrum. The blue is the residue, i.e. the difference between the experimental and the literature spectra.

\section{Calibration, performance and multi-species detection}

\subsection{Performance}

\subsubsection{Long-term stability and instrument sensitivity}

The same analysis proposed in the main manuscript for the IBBCEAS-NO ${ }_{2}$ are here also reported for the twin $I B B C E A S-\mathrm{NO}_{\mathrm{x}}$ instrument. The analysis were done under the same experimental conditions (instrument temperature of $12.0 \pm 0.2{ }^{\circ} \mathrm{C}$, cavity pressure of $630.0 \pm 0.7 \mathrm{mbar}$ and zero-air flow of $1.07 \pm 0.10 \mathrm{~L} \mathrm{~min}^{-1}$ ). Similar results on the minimum absorption coefficient and AW statistical analysis were obtained, proving the good reproductibility of those type of sensors. 


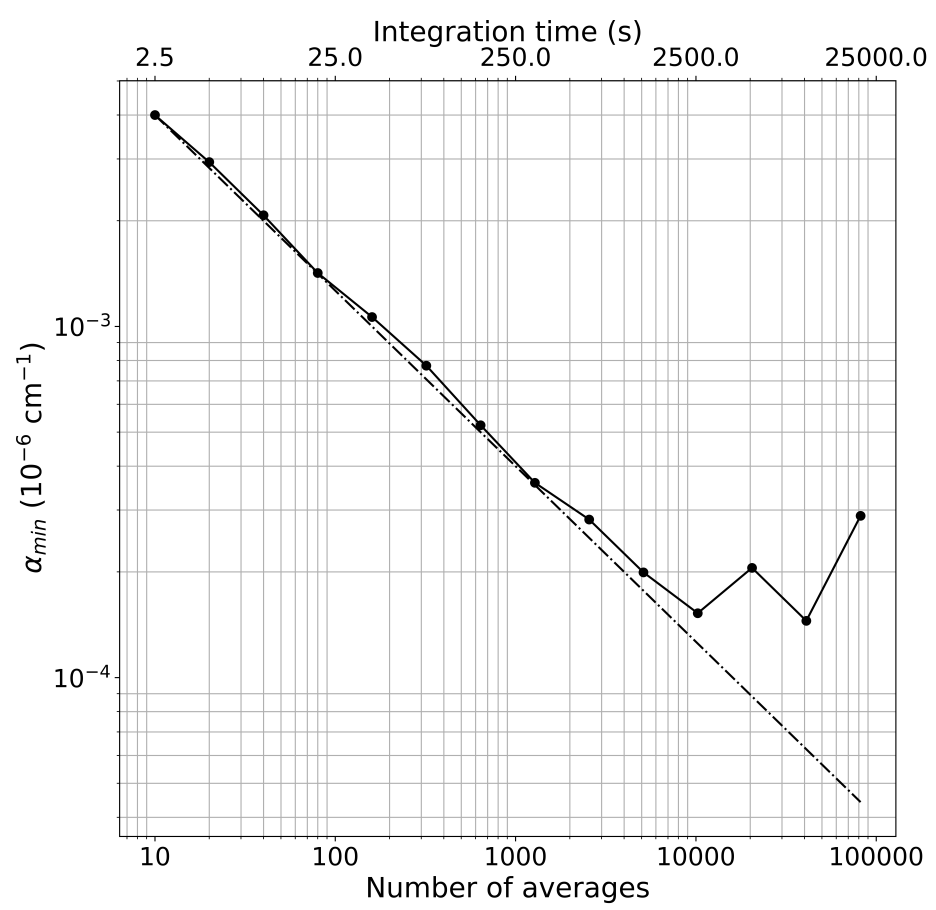

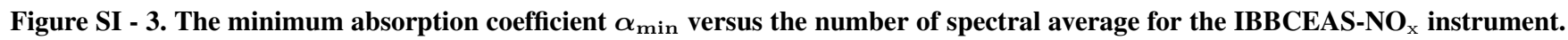
For these measurements the cell was continuously flushed with a flow of $1.07 \mathrm{~L} \mathrm{~min}{ }^{-1}$ of zero-air, and the $\alpha_{\text {min }}$ was calculated from the standard deviation of the residual of the spectra at different time averages.

Figure SI-4 shows FIT residuals retrieved using different number of averages (10, 5,120, 40,960 and 81,980). The more frequency noise is introduced, the more structures are observed on the residulas. It is therefore a question of finding the right number of averaging operations in order to use the instruments to the best of their respective abilities. 

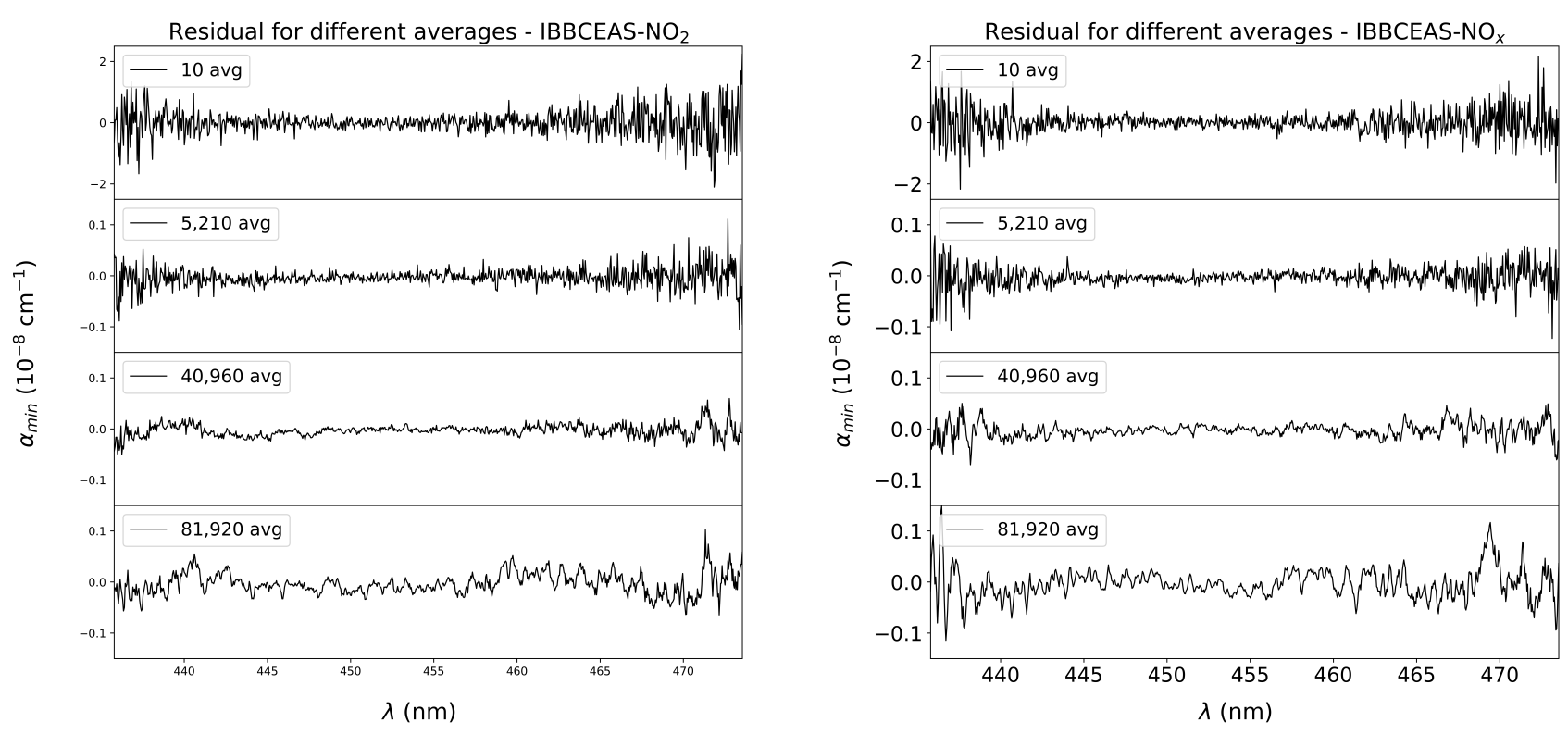

Figure SI - 4. Residuals of the spectral fit at different number of averages for the IBBCEAS-NO (left) and IBBCEAS-NO $_{x}$ instruments (right), showing the arise of structured frequency-dependent noise on the residual of the fit at larger averages.

For $2.5 \mathrm{~s}$ acquisition time, corresponding to an average of 10 spectra, the $\sigma_{\mathrm{AW}-\mathrm{SD}}$ was $210 \mathrm{ppt}$ for $\mathrm{NO}_{2}, 6.5 \mathrm{ppt}$ for IO, $180 \mathrm{ppt}$ for $\mathrm{CHOCHO}$ and $730 \mathrm{ppb}$ for $\mathrm{O}_{3}$. By averaging consecutive spectra, the $\sigma_{\mathrm{AW}-\mathrm{SD}}$ decreased following the white noise trend with a characteristic $\sqrt{N}$ slope (where $N$ is the number of spectra). The optimal sensitivity is achieved after 2,600 averages ( $\sim 11$ minutes of measurement for acquiring both the reference and the absorption spectra), where the $\sigma_{\mathrm{AW}-\mathrm{SD}}$ reaches 15 , $0.43,14 \mathrm{ppt}$ and $58 \mathrm{ppb}$ for $\mathrm{NO}_{2}, \mathrm{IO}, \mathrm{CHOCHO}$ and $\mathrm{O}_{3}(1 \sigma)$, respectively. If the optimum time of measurement is doubled, the system cand achieve ultimate detection limits of 12, 0.32, $11.5 \mathrm{ppt}$ and $48 \mathrm{ppb}$ for $\mathrm{NO}_{2}, \mathrm{IO}, \mathrm{CHOCHO}$ and $\mathrm{O}_{3}(1 \sigma)$, respectively. 

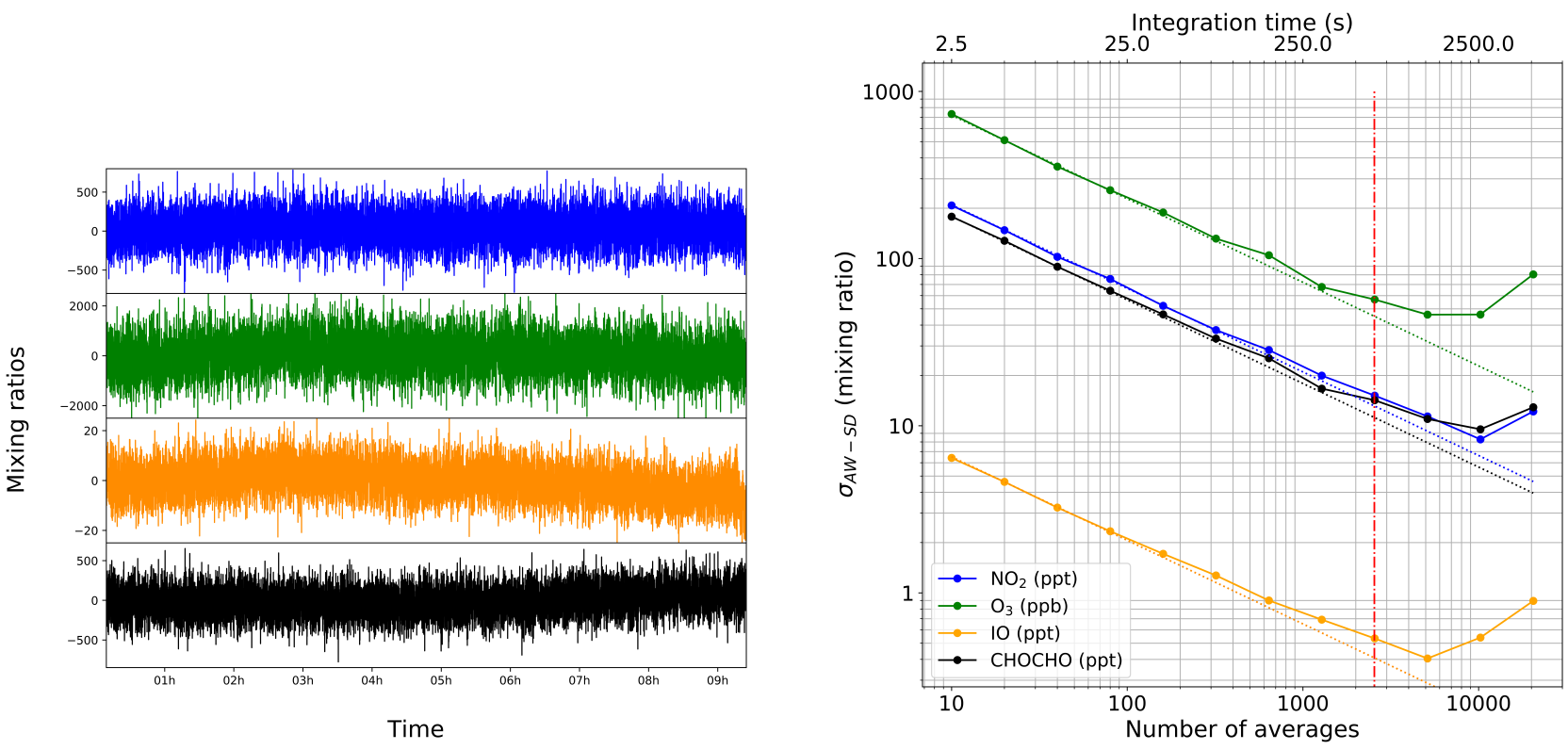

Figure SI - 5. (top) Mixing ratios of the target species $\mathrm{NO}_{2}, \mathrm{O}_{3}$, IO, and CHOCHO measured during a nine hours Allan-Werle vari-

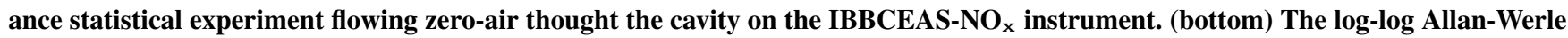
standard deviation plot, illustrating very similar behavior that for the IBBCEAS-NO $\mathrm{N}_{2}$ instrument reported in the main manuscript.

\subsection{Indirect measurement of NO}

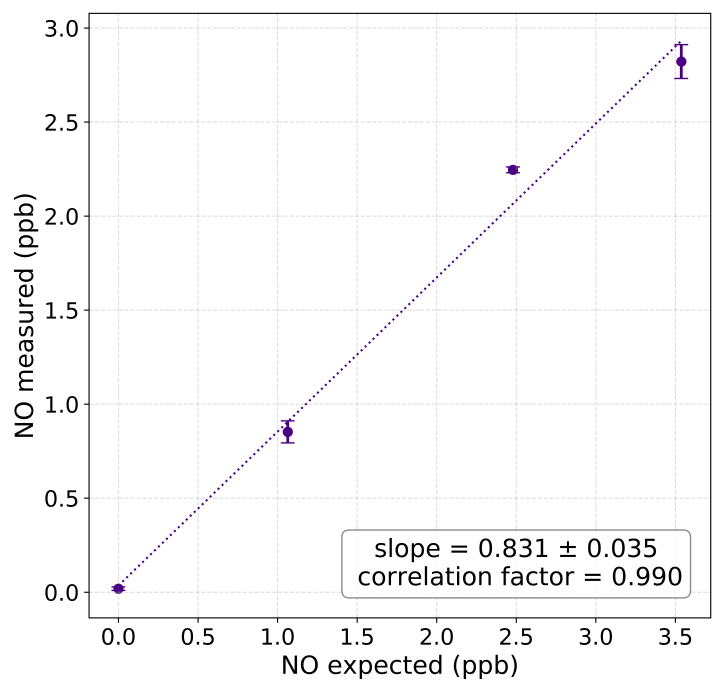

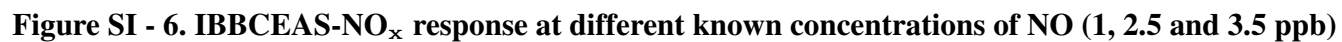


The response of the IBBCEAS-NOx instrument was tested by injecting different concentrations of $\mathrm{NO}(0,1,2.5$ and 3.5ppb). A $\mathrm{NO}$ cylinder from Air Liquide ( $\mathrm{NO}$ in $\mathrm{N}_{2}$ at $1 \mathrm{ppm} \pm 5 \%$ ) was used through a dilution line (Mass Flow Controllers from MKS instrument - ratio of 10,000:10) to perform the experiment. The cylinder was previously calibrated with a ThermoFisher ${ }^{\mathrm{TM}}$, model 42iTL trace analyzer. The results are shown in Figure SI-6 where a linear response with a slope of $0.831 \pm 0.035$ and a correlation factor $\mathrm{R}^{2}=0.990$ can be observed.

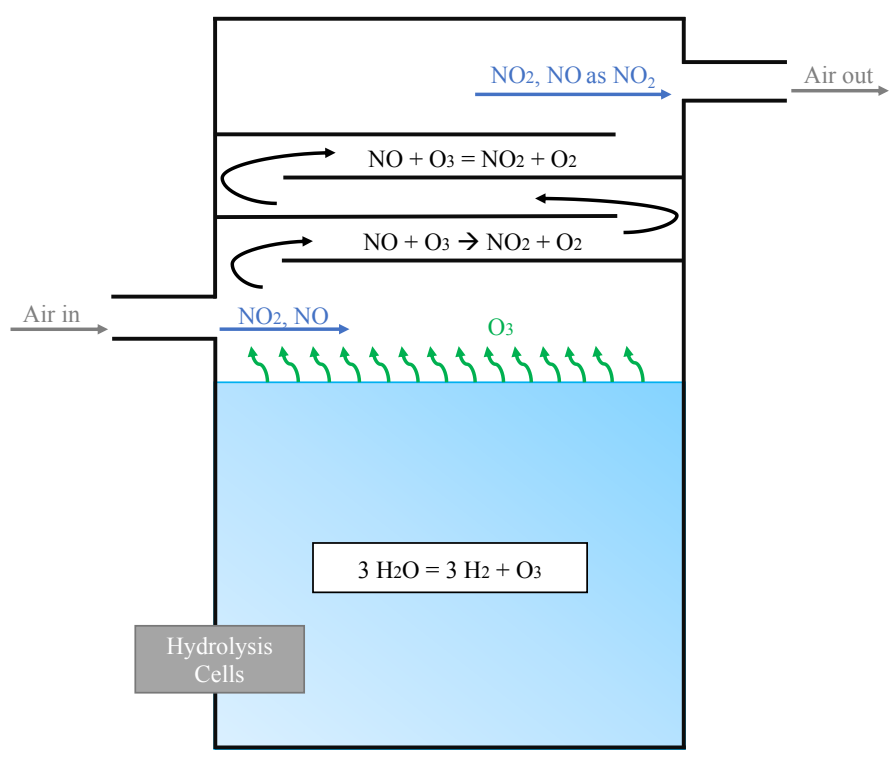

Figure SI - 7. Schematic design of the compact ozone generator. The system sizes is $15 \times 15$ x $15 \mathrm{~cm}^{3}$ with a water reservoir of 200 $\mathrm{cm}^{3}$. A series of electrolytic cells is placed at the bottom, and the air is flushed at the surface before being send to the spectrometer. The cover is designed for enhancing the contact between the water surface and the air as well as preventing water droplets to enter the outlet line.

The design of the container allows a maximization of the contact area between the air flow and the water and therefore a high ventilation at the surface. The cap was designed with a twisted channel for allowing a better mixing of the gas and preventing water droplet from entering the outlet line. 


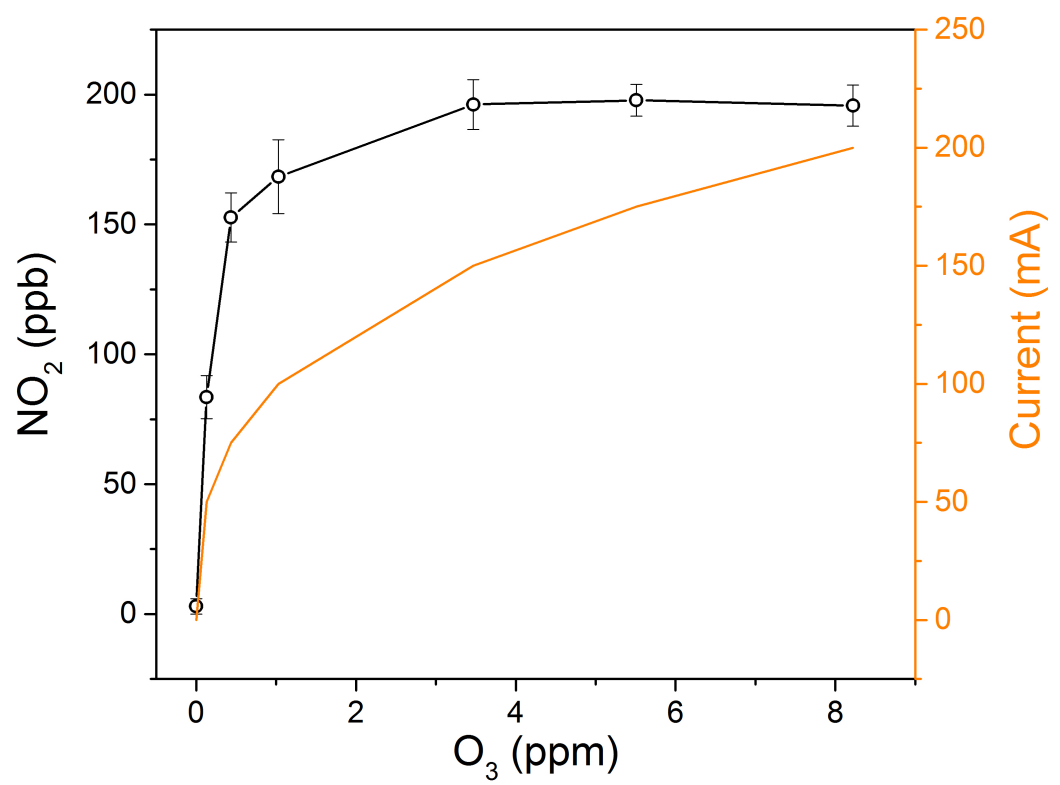

Figure SI - 8. The results from the conversion of $\mathrm{NO}$ to $\mathrm{NO}_{2}$ under ozone excess. The measured $\mathrm{NO}_{2}$ produced is plotted against the level of $\mathrm{O}_{3}$ produced by applying a certain current to the sequence of four electrolysis ozone-micro-cells. For the experiment a bottle of $\sim 180 \mathrm{ppb}$ of NO in air was used. 